\title{
Phytoprotection
}

phytoprotection

\section{Liste des arbitres 1999-2001}

\section{List of Reviewers 1999-2001}

Volume 82, numéro 3, 2001

URI : https://id.erudit.org/iderudit/706223ar

DOI : https://doi.org/10.7202/706223ar

Aller au sommaire du numéro

Éditeur(s)

Société de protection des plantes du Québec (SPPQ)l

ISSN

0031-9511 (imprimé)

1710-1603 (numérique)

Découvrir la revue

Citer ce document

(2001). Liste des arbitres 1999-2001. Phytoprotection, 82(3), 137-139.

https://doi.org/10.7202/706223ar d'utilisation que vous pouvez consulter en ligne.

https://apropos.erudit.org/fr/usagers/politique-dutilisation/ 
Le comité de rédaction de PHYTOPROTECTION veut reconnaître l'excellente contribution de nombreux collaborateurs qui ont généreusement participé au processus d'évaluation des manuscrits. Qu'ils trouvent ici l'expression de nos remerciements les plus sincères.

The Editorial Board of PHYTOPROTECTION would like to acknowledge the excellent contribution of many collaborators who generously participated in the evaluation of manuscripts. May they find here the expression of our most sincere gratitude.

Alford, A.

Arnason, J.T.

Baines, $P$.

Bartz, J.

Bélair, G.

Benoît, D.L.

Berger, R.

Bernier, L.

Boisclair, J.

Boiteau, G.

Brodeur, J.

Carroll, A.

Carroll, R.B.

Chen, $\mathrm{S}$.

Christie, B.

Cloutier, C.

Cossentine, J.

D'Arcy, C.

Desjardins, $Y$.

Dostaler, D.

Dumas, M.T.

Elliott, M.

Fenn, $\mathrm{P}$.
University of Maine, Orono, Maine

University of Ottawa, Ottawa, Ontario

Alberta Agriculture, Edmonton, Alberta

University of Florida, Gainesville, Florida

Agriculture et Agroalimentaire Canada, Saint-Jean-sur-Richelieu, Québec

Agriculture et Agroalimentaire Canada, Saint-Jean-sur-Richelieu, Québec

University of Florida, Gainesville, Florida

Université Laval, Québec, Québec

Institut de recherche et de développement en agroenvironnement, Saint-Hyacinthe, Québec

Agriculture et Agroalimentaire Canada, Frédéricton, Nouveau-Brunswick

Université Laval, Québec, Québec

Canadian Forest Service, Victoria, British Colombia

University of Delaware, Newark, Delaware

University of Minnesota, Waseca, Minnesota

Agriculture and Agri-Food Canada, Charlottetown, Prince Edward Island

Université Laval, Québec, Québec

Agriculture and Agri-Food Canada, Summerland, British Colombia

University of Illinois, Urbana, Illinois

Université Laval, Québec, Québec

Université Laval, Québec, Québec

Service canadien des forêts, Sault Sainte-Marie, Ontario

University of Florida, Gainesville, Florida

University of Arkansas, Arkansas 
Fitzpatrick, S.

Galvaski, J.

Gaudet, D.

Giblin-Davis, R.

Goettel, M.

Gold, J.

Goyer, C.

Grafius, E.D.

Hafez, S.

Herschman, D.E.

Hogue, R.

Holliday, N.

Innes, L.

Jacobi, B.

Kimbrough, J.

Kokalis-Burelle, N.

Kommedahl, $\mathrm{T}$.

Krupinski, J.

Kucharek, T.A.

Lamey, H.A.

Lazarovits, G.

Lemieux, C.

Lesage, $L$.

Liburd, $\mathrm{O}$.

Lucarotti, C.

MacGuidwin, A.E.

Maund, C.

McKenry, M.V.

McLachlan, S.

Mitchell, E.

Morin, B.

Nealis, V.

Nguyen, K.B.

Nicole, $M$.

Nie, $X$.
Agriculture and Agri-Food Canada, Agassiz, British Colombia

Manitoba Agriculture, Carmen, Manitoba

Agriculture and Agri-Food Canada, Lethbridge, Alberta

University of Florida, Ft. Lauderdale, Florida

Agriculture and Agri-Food Canada, Lethbridge, Alberta

Agriculture and Agri-Food Canada, Winnipeg, Manitoba

Agriculture et Agroalimentaire Canada, Frédéricton, Nouveau-Brunswick

Michigan State University, E. Lansing, Michigan

University of Idaho, Parma, Idaho

University of Kentucky, Princeton, Kentucky

Institut de recherche et de développement en agroenvironnement, Sainte-Foy, Québec

University of Manitoba, Winnipeg, Manitoba

Ministère des Ressources naturelles du Québec, Québec

Colorado State University, Ft. Collins, Colorado

University of Florida, Gainesville, Florida

USDA, Ft. Pierce, Florida

University of Minnesota, St.Paul, Minnesota

USDA, Manda, North Dakota

University of Florida, Gainesville, Florida

North Dakota State University, Fargo, North Dakota

Agriculture and Agri-Food Canada, London, Ontario

Agriculture et Agroalimentaire Canada, Sainte-Foy, Québec

Agriculture et Agroalimentaire Canada, Ottawa, Ontario

Michigan State University, E. Lansing, Michigan

Canadian Forest Service, Fredericton, New Brunswick

University of Wisconsin, Madison, Wisconsin

New Brunswick Agriculture \& Fisheries, Fredericton, New Brunswick

University of California, Parlier, California

University of Manitoba, Winnipeg, Manitoba

University of Florida, Gainesville, Florida

Atlantic Forestry Service, Fredericton, New Brunswick

Canadian Natural Resources, Victoria, British Colombia

University of Florida, Gainesville, Florida

IRD, Montpellier, France

Agriculture and Agri-Food Canada, Fredericton,

New Brunswick 
Nundt, C. Oregon State University, Corvallis, Oregon

Nyczepir, A.P. USDA, Byron, Georgia

Olfort, $\mathrm{O}$.

Agriculture and Agri-Food Canada, Saskatoon, Saskatchewan

Osborn, W. Pest Management Regulatory Service, Ottawa, Ontario

Pelletier, Y. Agriculture et Agroalimentaire Canada, Frédéricton, Nouveau-Brunswick

Peters, R.

Agriculture and Agri-Food Canada, Charlottetown, Prince Edward Island

Potter, J.

Agriculture and Agri-Food Canada, Vineland, Ontario

Powers, T.O.

University of Nebraska, Lincoln, Nebraska

Pratt, $P$.

Oklahoma State University, Oklahoma

Quiring, D.

University of New Brunswick, Fredericton, New Brunswick

Rich, J.R.

University of Florida, Quiney, Florida

Rochon, D.

Agriculture and Agri-Food Canada, Summerland, British Colombia

Ronis, D.

McCain Produce, Florenceville, New Brunswick

Royer, L.

Canadian Forest Service, Corner Brook, New Foundland

Santo, G.S. Washington State University, Prosser, Washington

Saint-Arnaud, M. Institut de recherche en biologie végétale, Montréal, Québec

Secor, G.

North Dakota State University, Fargo, North Dakota

Stiles, C.

University of Florida, Gainesville, Florida

Tardif, F.

University of Guelph, Guelph, Ontario

Thistlewood, $\mathrm{H}$.

Agriculture and Agri-Food Canada, Summerland, British Colombia

Thurston, G. $\quad$ Canadian Forest Service, Fredericton, New Brunswick

Todd, T.C.

Kansas State University, Manhattan, Kansas

Trimble, $\mathrm{M}$.

Agriculture and Agri-Food Canada, Vineland, Ontario

Turkington, $\mathrm{K}$.

Agriculture and Agri-Food Canada, Lacombe, Alberta

Weingardner, $P$.

University of Florida, Hastings, Florida

Williamson, $\mathrm{V}$.

University of California, Davis, California

Wise, I.

Agriculture and Agri-Food Canada, Winnipeg, Manitoba

Yuen, G.

University of Nebraska, Lincoln, Nebraska 